\title{
Questionable Conclusions?
}

\author{
Rachel Perrya, ${ }^{\mathrm{a}}$, Richard Makins ${ }^{\mathrm{a}}$
}

\section{To the Editor}

I took a great interest in the paper entitled "Lactic Acid Is an Independent Predictor of Mortality and Improves the Predictive Value of Existing Risk Scores in Patients Presenting With Acute Gastrointestinal Bleeding" published in the February edition of Gastroenterology Research. As a hospital doctor regularly admitting patients presenting with acute gastrointestinal (GI) bleeding, I can see significant benefits with regards to triaging and managing such patients using lactic acid measurements as a predictor of mortality. However, I am concerned about the validity of the conclusions that the authors have drawn.

The authors have used venous lactic acid measured within $24 \mathrm{~h}$ of admission to hospital. While the authors acknowledge that there may be some selection bias regarding which patients have this measurement completed (and are therefore included into the study), I do not feel there is adequate recognition as to what stage of resuscitation the patient has had this measurement done. A patient's lactic acid measured within a few minutes of admission is likely to be much greater than an equivalent measurement done after $24 \mathrm{~h}$ of resuscitation, which would create significant bias in the results.

As the authors acknowledge, of the 366 patients admitted with an acute upper or lower GI bleed, only 188 had an endoscopy. Of particular note, only 14 of the 30 patients who did not survive their inpatient admission had an endoscopy. While I appreciate this was a retrospective study and that there are many reasons why patients do not proceed to invasive investigation, I feel that $51 \%$ of patients is too low of value without explanation as to why this was the case. Were patients who were labelled as having an acute GI bleed on triage later considered to have an alternative diagnosis? If so, I question whether these patients should be included in the statistical analysis. Furthermore, $23.6 \%$ of survivors and $14.3 \%$ of nonsurvivors who did have an endoscopy did not have any stigmata of bleeding seen on endoscopy. Although these patients' serum results and clinical picture may be convincing for a GI bleed from a source not seen on endoscopy, is it too high a proportion of patients to label as having had an acute GI bleed and subsequently include in the statistical analysis, without explanation? These points lead me to doubt what proportion of

Manuscript submitted April 18, 2019, accepted August 5, 2019

${ }^{a}$ Gloucestershire Hospitals NHS Foundation Trust, Gloucester, UK

${ }^{\mathrm{b} C o r r e s p o n d i n g ~ A u t h o r: ~ R a c h e l ~ P e r r y, ~ G l o u c e s t e r s h i r e ~ H o s p i t a l s ~ N H S ~ F o u n-~}$ dation Trust, Gloucester, UK. Email: rachelsarah.perry@nhs.net the 366 patients did have an acute GI bleed, and therefore the evidence for using lactic acid measurements to predict mortality in this patient population.

Another concern I have is that the authors conclude that lactic acid can be used as a predictor of mortality in unselected bleeds, yet do not demonstrate that their results are statistically significant for both upper and lower GI bleeds independently. This is significant given the known greater burden of mortality with acute upper GI bleeds compared with acute lower GI bleeds. With no indication of what proportion of patients who were nonsurvivors had lower GI bleeds, can the authors confidently extrapolate their findings in such a way that has been done?

In conclusion, I believe that there are oversights in the methodology of this paper, which would question the conclusion that lactic acid is both an independent predictor of mortality and improves the predictive value of existing risk scores in unselected acute GI bleeds.

\section{Acknowledgments}

Not applicable.

\section{Financial Disclosure}

Not applicable.

\section{Conflict of Interest}

None to declare.

\section{Informed Consent}

Not applicable.

\section{Author Contributions}

Rachel Perry: writing of the letter. Richard Makins: proofreading of the letter.

\section{Reply From Dr. Getu Teressa et al}

We thank Perry and Makins for their comments. We agree 
with their point that the value of the lactic acid measured can be significantly affected by resuscitation efforts. As such, this underscores a limitation of the retrospective study whereby lactic acid was not uniformly measured in all patients at any specified point during resuscitation effort, nor were there serial measurements in all patients to enable the evaluation of the trend and capture the actual peak lactic acid value. Despite this and several limitations specified in the manuscript, we believe our study complement several other retrospective studies [1-7] and collectively should provide an impetus for a prospective study. In agreement with Perry and Makins, we believe the timing of lactic acid measurement in relation to resuscitation should be taken into consideration in any future prospective study.

Perry and Makins stated that the rate of endoscopic evaluations in the study, which was about $51 \%$, was "too low" and wondered why this was the case. We do believe this rate is not low considering the design of the study, epidemiology of acute gastrointestinal bleeding and variable clinical course of patients after initial presentation to the emergency department (ED) with "acute GI bleed". Our research design was in recognition of an unmet need for validated tools for frontline clinicians to risk-stratify patients as they arrive in the ED with GI bleeding. Therefore, as part of our research design, our cohort included all patients with acute GI bleeding on initial presentation to the ED, regardless of the clinically suspected site of upper vs. lower GI bleed or subsequent course of the GI bleed (self-limited or sustained). Although we did not ascertain which fraction of the cohort had clinically suspected upper GI bleed (by reviewing charts), the $51 \%$ rate of endoscopic evaluations in our cohort likely reflects the proportion of those with suspected upper GI source for their bleeding. This rate mirrors the results of the largest population-based study that showed upper GI bleeding was responsible for about $51 \%$ of hospitalization for GI Bleed or preformation events. Additionally, this study demonstrated an impressive decreasing temporal trend in upper GI bleed between 1996 and 2005 [8]. Therefore, we believe the $51 \%$ rate of endoscopy among patients presenting to the ED with acute GI bleed is not low, but rather in line with the overall epidemiology/prevalence of upper GI bleeding from external studies. To the contrary, taking into account the declining trend in upper GI bleed presentation between 1996 and 2005 [8], our study may suggest a liberal utilization of endoscopic evaluation in a contemporary patient population presenting with GI bleed, with a projected lower prevalence of upper GI bleed than two decades ago.

Perry and Makins also pointed out that the proportion of patients found to have "no lesion or stigmata of recent bleed" was "too high" (23.6\% of survivors and $14.3 \%$ non-survivors). As one could appreciate, not all endoscopic examinations are expected to yield positive or relevant findings, even in the setting of acute GI bleeding. Although the authors did not cite a reference as a basis for this question or what is deemed an acceptable rate of negative findings, they implicitly raised crucial questions regarding the diagnostic yield of endoscopy in contemporary patients evaluated for suspected acute upper GI bleed and if there is an acceptable threshold/benchmark for diagnostic yield rate.

In a recent meta-analysis of 23 studies comprising a total of 53,392 patients, the prevalence of relevant findings on upper GI endoscopy was a little more than 50\% [9]. There are very limited data from large studies on the yield of endoscopy in patients with suspected upper GI bleed. A small study comprising of patients with suspected upper GI bleeding (similar to ours), negative endoscopic evaluation was observed in $24.6 \%$ of patients [10]. Even among critically ill patients admitted to the intensive care unit who underwent bedside endoscope for suspected GI bleed, the diagnostic yield was found to be 82 $85 \%$ (i.e. $15-18 \%$ endoscopies with no identifiable source) [11, 12]. In the absence of a defined benchmark for an acceptable rate of diagnostic yield, we can only say that the diagnostic yield of the endoscopic examinations in our study is within the range of current prevailing practices.

One explanation for normal finding in the setting of acute GI bleed is possible errors in the clinical impression of the site of GI bleeding at an earlier point in the evaluation. As discussed in our manuscript, we speculated that the limited application of existing risk scores, which are specifically developed for either upper or lower GI bleed, is due to uncertainties in discerning upper vs. lower GI bleed by frontline providers. There are no studies to our knowledge that assessed the degree of correlation between initial clinicians' impression of the sites of bleeding and the confirmed sites of bleeding after complete evaluation. The prospective study by Laine and Shah reinforces this uncertainty in clinical practice by demonstrating that $15 \%$ of patients who were initially presumed to have lower GI bleed were ultimately found to have an upper GI source for their bleeding [13].

We thank Perry and Makins for their comments, and we cannot agree more with their conclusion that there is not sufficient evidence yet to use lactic acid in clinical decision making in patients with an acute GI bleed. Nonetheless, our study was overwhelmingly in agreement with several previous observational retrospective studies [1-7], and we hope there is sufficient momentum for a prospective study to evaluate the value of incorporating lactic acid in risk stratification of patients with acute GI bleed in such a way that it proves useful for frontline care providers.

Getu Teressa, MD, PhD

Assistant Professor of Clinical Medicine, Stony Brook Medicine, Stony Brook, NY, USA

Matthew Berger, MD

Internal Medicine Resident, Stony Brook Medicine, Stony Brook, NY, USA

Vadim Divilov, MD

Internal Medicine Resident, Stony Brook Medicine, Stony Brook, NY, USA

\section{References}

1. El-Kersh K, Chaddha U, Sinha RS, Saad M, Guardiola J, Cavallazzi R. Predictive role of admission lactate level in critically ill patients with acute upper gastrointestinal bleeding. J Emerg Med. 2015;49(3):318-325.

2. Ko BS, Kim WY, Ryoo SM, Ahn S, Sohn CH, Seo DW, Lee YS, et al. Predicting the occurrence of hypotension 
in stable patients with nonvariceal upper gastrointestinal bleeding: point-of-care lactate testing. Crit Care Med. 2015;43(11):2409-2415.

3. Kwon YH. Lactate parameters predict clinical outcomes in patients with nonvariceal upper gastrointestinal bleeding. J Korean Med Sci. 2017;32(11):1731.

4. Lee SH, Min YW, Bae J, Lee H, Min BH, Lee JH, Rhee PL, et al. Lactate parameters predict clinical outcomes in patients with nonvariceal upper gastrointestinal bleeding. J Korean Med Sci. 2017;32(11):1820-1827.

5. Shah A, Chisolm-Straker M, Alexander A, Rattu M, Dikdan S, Manini AF. Prognostic use of lactate to predict inpatient mortality in acute gastrointestinal hemorrhage. Am J Emerg Med. 2014;32(7):752-755.

6. Shrestha MP, Borgstrom M, Trowers EA. Elevated lactate level predicts intensive care unit admissions, endoscopies and transfusions in patients with acute gastrointestinal bleeding. Clin Exp Gastroenterol. 2018;11:185-192.

7. Wada T, Hagiwara A, Uemura T, Yahagi N, Kimura A. Early lactate clearance for predicting active bleeding in critically ill patients with acute upper gastrointestinal bleeding: a retrospective study. Intern Emerg Med. 2016;11(5):737-743.

8. Lanas A, Garcia-Rodriguez LA, Polo-Tomas M, Ponce
M, Alonso-Abreu I, Perez-Aisa MA, Perez-Gisbert J, et al. Time trends and impact of upper and lower gastrointestinal bleeding and perforation in clinical practice. Am J Gastroenterol. 2009;104(7):1633-1641.

9. Zullo A, Manta R, De Francesco V, Fiorini G, Hassan C, Vaira D. Diagnostic yield of upper endoscopy according to appropriateness: A systematic review. Dig Liver Dis. 2019;51(3):335-339.

10. Dolmans WM, Mbaga IM, Mwakyusa DH. Diagnostic yield of endoscopy in upper gastrointestinal bleeding. Trop Geogr Med. 1983;35(2):173-178.

11. Kim JH, Kim JH, Chun J, Lee C, Im JP, Kim JS. Early versus late bedside endoscopy for gastrointestinal bleeding in critically ill patients. Korean J Intern Med. 2018;33(2):304-312.

12. Lee YC, Wang HP, Wu MS, Yang CS, Chang YT, Lin JT. Urgent bedside endoscopy for clinically significant upper gastrointestinal hemorrhage after admission to the intensive care unit. Intensive Care Med. 2003;29(10):17231728.

13. Laine L, Shah A. Randomized trial of urgent vs. elective colonoscopy in patients hospitalized with lower GI bleeding. Am J Gastroenterol. 2010;105(12):2636-2641; quiz 2642 . 